\title{
THE RICCATI FLOW AND SINGULARITIES OF SCHUBERT VARIETIES
}

\author{
JAMES S. WOLPER
}

(Communicated by Louis J. Ratliff, Jr.)

\begin{abstract}
Let $\operatorname{Gr}(m, n)$ be the Grassmannian of $m$-dimensional subspaces of an $n$-dimensional $k$-vector space, with $k=\mathbf{R}$ or $\mathbf{C}$. Fix an $n \times n$ matrix $R$ with coefficients in $k$. The Riccati Flow $\Phi$ on $\operatorname{Gr}(m, n)$ is the action of a one-parameter subgroup of $\mathrm{GL}_{n}(k)$, given by $\Phi_{t}(\Lambda)=e^{R t} \Lambda$. We prove:
\end{abstract}

Theorem. Let $X$ be a Schubert variety in $\operatorname{Gr}(m, n)$. Then there exists a Riccati flow $\Phi$ on $X$ and a stable manifold $W$ for $\Phi$ such that $W$ is the smooth locus of $X$.

Corollary (over C). $X$ as above is smooth if and only if the cohomology of $X$ satisfies Poincaré Duality.

\section{INTRODUCTION}

Schubert varieties and their singularities have been a focus of recent research in algebraic topology, algebraic geometry, representation theory, combinatorics, and systems and control theory (see [GM] for references). There are several ways to detect singularities: briefly, these are Kazhdan-Lusztig polynomials (representation theory), intersection cohomology (algebraic topology), Standard Monomial Theory (algebraic geometry), and "lexicographically" (combinatorics and algebraic geometry). This paper presents a new approach to the computation of the singular locus of a Schubert variety in a Grassmannian.

The basic tool in this approach is a certain one-parameter subgroup of the general linear group, called the Riccati flow; the reason for the name will be discussed below.

In the following, $k$ denotes either the real or complex numbers; the Grassmannian $\operatorname{Gr}(m, n)$ is the variety of $m$-dimensional subspaces of a vector space of dimension $n$ over $k$; and cohomology is singular cohomology with integral coefficients, unless explicitly stated otherwise.

The main result is

(1.1) Theorem. Let $X$ be a Schubert variety in $\operatorname{Gr}(m, n)$. Then there exists a Riccati flow on $\operatorname{Gr}(m, n)$ and a stable manifold $W$ of this flow such that $W$ is exactly the smooth locus of $X$.

Received by the editors September 12, 1990 and, in revised form, June 6, 1993; presented at AMS meeting \#849, Chicago, IL, May 1989.

1991 Mathematics Subject Classification. Primary 14M15. 
This has the following interesting corollary, which should be compared with a result proved for general Schubert varieties by Deodhar [D] using Standard Monomial Theory. The present proof does not, however, use the representation theory of $\mathrm{SL}_{n}$.

(1.2) Corollary (over C). Let $X$ be a Schubert variety in $\operatorname{Gr}(m, n)$. Then $X$ is smooth if and only if the singular cohomology of $X$ satisfies Poincaré Duality.

The contents are a brief review of Schubert varieties ( $\$ 2)$, Shayman's phase portrait for the Riccati flow $(\S 3)$, and the proofs $(\S 4)$.

Some of this work was completed while the author was a Visiting Assistant Professor at Texas Tech University, whose support and hospitality are gratefully acknowledged.

\section{Review of Schubert CElls AND Schubert VARIETIES}

This section presents a brief review of Schubert cells and Schubert varieties. See $[\mathrm{W}]$ or $[\mathrm{GeM}]$ for details.

The Schubert cells form a cell decomposition of the Grassmannian $\operatorname{Gr}(m, n)$ and are defined as follows. Let $V_{n}$ be a vector space of dimension $n$ over $k$. Fix a complete flag of subspaces

$$
F_{1} \subset F_{2} \subset \cdots \subset F_{n}=V_{n},
$$

where $\operatorname{dim}\left(F_{i}\right)=i$. Choose $m$ integers $d_{1}<\cdots<d_{m} \leq n$. Define the Schubert cell $\left[d_{1} d_{2} \cdots d_{m}\right]$ by

$$
\left[d_{1} \cdots d_{m}\right]=\left\{\Lambda \in \operatorname{Gr}(m, n): \operatorname{dim}\left(\Lambda \cap F_{d_{j}}\right)=j, \operatorname{dim}\left(\Lambda \cap F_{d_{j}}\right) \neq j+1\right\} .
$$

The closure of $\left[d_{1} \cdots d_{m}\right]$ in $\operatorname{Gr}(m, n)$ is a Schubert variety; it is a complete irreducible projective variety of $k$-dimension $\Sigma\left(d_{j}-j\right)$. It is a fundamental fact that a Schubert variety is a disjoint union of Schubert cells.

A Schubert cell (or variety-context makes clear which is meant) can be represented by a word of length $n$ constructed from the alphabet $\{a, b\}$. The letters in positions $d_{j}, j=1, \ldots, m$, are $a$, while the remaining letters are $b$. The string $a a \cdots a$ ( $k$ occurrences) is often abbreviated $a^{k}$. Thus, for example, the unique codimension 1 Schubert variety in $\operatorname{Gr}(2,4)$ can be represented as [24] or by the word baba. It consists of those $\Lambda$ which satisfy the two "Schubert conditions"

and

$$
\operatorname{dim}\left(\Lambda \cap F_{2}\right)=1, \quad \Lambda \neq F_{2},
$$

$$
\operatorname{dim}\left(\Lambda \cap F_{4}\right)=2, \quad \text { i.e., } \Lambda \subset F_{4} .
$$

(Although the last condition is, in fact, vacuous.)

There is a partial order on the set of Schubert cells in $\operatorname{Gr}(m, n)$, variously called the Bruhat order, the adherence order, or the specialization order (see [P] or [HM]). The easiest way to define it for Schubert cells $C_{1}$ and $C_{2}$ is by

$$
C_{1} \leq C_{2} \Leftrightarrow C_{1} \subset \bar{C}_{2},
$$

where $\bar{X}$ denotes the closure of $X$. This order is generated by the "moves"

$$
w_{1} b a w_{2}>w_{1} a b w_{2} \text {, }
$$

where $w_{1}$ and $w_{2}$ are arbitrary subwords. See [P]. 
The cells with this partial order define a lattice, called the Hasse diagram. For $\operatorname{Gr}(2,4)$ it is

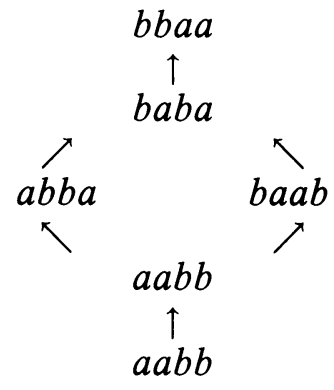

where $w \rightarrow w^{\prime}$ indicates that $w \subset \overline{w^{\prime}}$.

\section{THE RiCCATI FLOW}

Let $R$ be an $(n+m) \times(n+m)$ matrix with entries in $k, k=\mathbf{C}$ or $k=\mathbf{R}$. The Riccati Flow $\Phi$ generated by $R$ on $\operatorname{Gr}(n, n+m)$ is given by

$$
\Phi_{t}(\Lambda)=e^{R t} \Lambda
$$

where $\Lambda \in \operatorname{Gr}(n, n+m), e^{R t}$ is the matrix exponential, $t \in k$, and the righthand side is the usual action of $\mathrm{GL}(n+m)$ on subspaces. The name comes from systems and control theory: there are local coordinates on the Grassmannian such that the flow is infinitesimally generated by the vector field

$$
\frac{d K}{d t}=R_{21}+R_{22} K-K R_{11}-K R_{12} K
$$

when the block decomposition for $R$ is

$$
\left(\begin{array}{ll}
R_{11} & R_{12} \\
R_{21} & R_{22}
\end{array}\right) .
$$

This is the matrix Riccati equation, which is used in certain optimal control problems [B]. The flow can be extended to various flag manifolds in an obvious manner.

Control theorists are most interested in the case of a semisimple $R$ whose eigenvalues have distinct real parts except those that occur as pairs of complex conjugates. Shayman [S] determined the phase portrait for the Riccati equation on the Grassmannian for semisimple $R$. (Although he worked over $\mathbf{R}$, the same proofs go through for $\mathbf{C}$ with minor modifications.) This involves finding what [S] refers to as the various invariant loci $T_{1}, \ldots, T_{r}$ and stable manifolds

$$
W_{i}=\left\{\Lambda: \lim _{t \rightarrow \infty} \Phi_{t} \Lambda \in T_{i}\right\}
$$

When $R$ is semisimple, the flow is a $C^{*}$ action on the Grassmannian.

(More generally, recent work of the author indicates that that these results follow from the Schur-Kostant-Atiyah-Guillemin-Sternberg Convexity Theorem [A]. Also, compare this with the Bialynicki-Birula plus and minus decompositions for a scheme with torus action [BB]. Note that in the proof of (1.1) we will construct an $R$ with the desired properties, so the extra assumptions are justified.) 
Here are Shayman's results. Fix an $(n+m)$-dimensional $k$-vector space $V$. Let $\mu_{1}<\cdots<\mu_{r}$ be the real parts of the eigenvalues of $R$. Then $V$ has the direct sum decomposition

$$
V=E_{1} \oplus \cdots \oplus E_{r}
$$

where each $E_{j}$ is the direct sum of the primary components of $R$ corresponding to those eigenvalues with real part $\mu_{i}$; let $d_{i}$ be the dimension of $E_{i}$. Clearly $E_{i}$ is $R$-invariant. Let $\left(l_{1}, \ldots, l_{r}\right)$ be a partition of $n$ with $l_{j} \leq d_{j}$. Define

$$
M_{j}=E_{1} \oplus \cdots \oplus E_{j}
$$

and

$$
T(l)=\operatorname{Gr}\left(l_{1}, E_{1}\right) \times \cdots \times \operatorname{Gr}\left(l_{r}, E_{r}\right) .
$$

(3.1) Theorem (Shayman). (i) For all $l$ as above, $T(l)$ is invariant with respect to the Riccati flow;

(ii) its stable manifold is

$$
W(l)=\left\{\Lambda \in \operatorname{Gr}(n, n+m): \operatorname{dim}\left(\Lambda \cap M_{s}\right)=\sum_{j=1}^{s} l_{j}, s=1, \ldots, r\right\} .
$$

(3.2) Proposition. $W(l)$ is dense in the Schubert variety whose word is $w=$ $w_{1} w_{2} \cdots w_{r}$, where $w_{j}=b^{d_{j}-l} a^{l_{j}}$, and $\overline{W(l)}=\bar{w}$.

Proof. We construct the word for the largest cell in $W(l)$; the proposition follows immediately.

The word decomposes into subwords $w_{j}$, each corresponding to the conditions imposed on an $E_{j}$. Let $s=\operatorname{dim}\left(M_{j}\right)$. If $\operatorname{dim}\left(E_{j}\right)=1=l_{1}$, the $j$ th Schubert condition on $\Lambda$ is $\operatorname{dim}\left(\Lambda \cap F_{s}\right)=1$, so $w_{j}=a$. Similarly if $l_{j}=0$, $w_{j}=b$. If $\operatorname{dim}\left(E_{j}\right)=2$ and $l_{j}=0$, the $j$ th condition is $\operatorname{dim}\left(\Lambda \cap F_{s}\right)=0$, so $w_{1}=b b$; if $l_{j}=1, w_{1}=b a$; and if $l_{j}=2, w_{1}=a a$. Notice that if $l_{j}=1$, there is an apparent ambiguity, because the subword $a b$ (corresponding to the condition $\left.F_{1} \subset \Lambda\right)$ also applies. However, the cell $a b w^{\prime}$ is in the closure of baw'. Q.E.D.

(3.3) Example. Let $r=3$, and choose $E_{j}$ such that $\operatorname{dim}\left(E_{j}\right)=2$ ( $j=$ $1,2,3)$, so $\operatorname{dim}\left(M_{1}\right)=2, \operatorname{dim}\left(M_{2}\right)=4$, and $\operatorname{dim}\left(M_{3}\right)=6$. Suppose $l=$ $(1,0,2)$. Then the $\Lambda$ in $W(l)$ satisfy the conditions $\operatorname{dim}\left(\Lambda \cap M_{1}\right)=1$, $\operatorname{dim}\left(\Lambda \cap M_{2}\right)=1$, and $\operatorname{dim}\left(\Lambda \cap M_{3}\right)=3$, and the word is babbaa. Similarly if $l=(1,1,1)$, the word is bababa.

\section{Proofs}

To begin, choose a Schubert variety $X$ in $\operatorname{Gr}(n, n+m)$. The proof of (1.1) involves constructing a partition $\left(d_{1}, \ldots, d_{n}\right)$ of $n+m$, constructing a matrix $R$ with primary components $E_{j}$ of dimension $d_{j}$, and choosing a sequence $\left(l_{1}, \ldots, l_{n}\right)$ of nonnegative integers with $l_{j} \leq d_{j}$.

Partition $n+m$ into $n$ integers as follows: let $w_{1}, \ldots, w_{n}$ be the subwords of the word for $X$ which end with $a$. For example, if $X=b b a a b a a$, then $n=4$ and $w_{1}=b b a, w_{2}=a, w_{3}=b a$, and $w_{4}=a$. Let $d_{j}$ be the number of letters in $w_{j}$. The desired partition is $\left(d_{1}, \ldots, d_{n}\right)$. 
Define the matrix $R$ with $n$ Jordan blocks of size $d_{j}$ having eigenvalue $-j$ (the actual value of the eigenvalue is not important; the choice here is just for reference). Let $E_{j}$ be the subspace of $k^{n+m}$ corresponding to the $j$ th block. Finally, let $l_{j}=1$, i.e., le $、 W=W(1, \ldots, 1)$. It is clear that the largest cell in $W$ is the largest cell in $X . W$ is the union of cells whose words consist of $n$ subwords $v_{j}$ of length $d_{j}$ containing a single $a$,

Informally, cells in $W$ are named by moving an $a$ within a subword but never by moving it into a different subword. When an $a$ moves into a different subword, the word so created represents a cell in the closure of $W$ but not in $W$. These cells are "just below" $W$ in the Hasse diagram. Call these cells "just below" $W$ the shear zone. For example, consider $W$ given by bababa. The shear zone consists of $a a b b b a$ and $b a a a b b$. The cell $a a b b a b$ is of codimension 1 in the closure of $a b a b a b$, a cell in the closure of bababa, and so might appear to be in the shear zone; however, it is already in the closure of a shear zone cell, e.g., aabbba.

This concept simplifies the statement of the main theorem:

(1.1, bis) Theorem. The singular locus of $X$ is the closure of the shear zone.

(4.1) Lemma (Characterization of the Shear Zone). The shear zone is the set of cells made up of $n$ subwords $v_{j}$ of length $d_{j}$, exactly one of which contains two occurrences of the letter $a$. In other words, cells in the shear zone have the form

$$
w_{1} w_{2} \cdots w_{s}^{\prime} w_{s+1}^{\prime} \cdots w_{n}
$$

where $w_{s}^{\prime}=b^{d_{s}-2} a a$ and $w_{s+1}^{\prime}=b^{d_{s+1}}$.

Thus, if $C$ is a cell in the shear zone, its word differs from that of $W$ in at most two subwords: the one with two as, and the one with no as.

We now proceed by induction on the dimension of $X$. It is trivial to check that all Schubert varieties of dimension 1 or 2 are nonsingular and, in these cases, that $W=X$. Similarly, there are four families of Schubert varieties of dimension 3: $a b b a B, b a a a B, b b b a B$, and $b a b a B$ (here, $B$ indicates a string of arbitrary length of the letter $b$ ). Members of the first three are nonsingular, and all satisfy $W=X$; those in the last are singular. The Hasse diagram of such an $X$ is

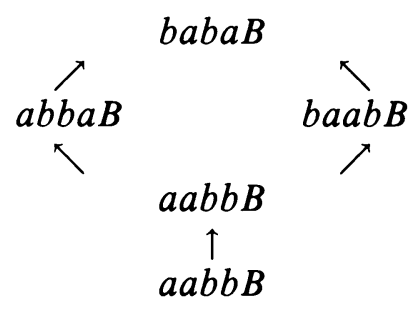

while $W$ consists of all these cells except $a a b b B$. It can be verified directly that $a a b b B$ is the singular locus. Thus, the theorem is true in the case of dimension 3 or less.

Now, restrict attention to the shear zone in the cell decomposition of $X$. If $n=2$, then there are two subwords. The shear zone consists of the cell $b^{d_{1}-2} a a b^{d_{2}}$. It is easy to verify directly that the closure of this cell is the singular locus (for example, write down a parametrization of $X$ ). 
If $n>3$, then the word for a cell in the shear zone differs from that of $W$ in. two subwords: the one with $a a$, and the one with no as. There must therefore exist, for each cell $C$ in the shear zone, an integer $r(0<r<n)$ such that one of the following conditions holds: or

(L) the first $r$ subwords of $C$ are identical to the first $r$ subwords of $W$;

(R) the last $r$ subwords of $C$ are identical to the last $r$ subwords of $W$.

Case $(\mathbf{R})$. Consider the fibering $\phi: \operatorname{Gr}(n, n+m) \rightarrow \operatorname{Gr}\left(n-1, n+m-d_{1}\right)$ given by

$$
\phi(\Lambda):=\left(\Lambda /\left(\Lambda \cap E_{1}\right) \subset\left(V / E_{1}\right)\right) ;
$$

in local coordinates, $\phi$ is a projection between vector spaces. Then $\phi(C)$ is in the shear zone of some stable manifold $W^{\prime}$ for the flow on $\operatorname{Gr}\left(n-1, n+m-d_{1}\right)$ corresponding to the lower right $\left(n+m-d_{1}\right) \times\left(n+m-d_{1}\right)$ submatrix of $R$; this follows immediately from the combinatorial characterization of the shear zone. Since the image cell is in the singular locus of the closure of $W^{\prime}$ and since $\phi$ is locally a projection, $C$ is in the singular locus of the closure of $W$.

Case (L). In this case, define

$$
E=E_{1} \oplus \cdots \oplus E_{n-1},
$$

and let $d=\operatorname{dim}(E)$. Define a map $\operatorname{Gr}(n, n+m) \rightarrow \operatorname{Gr}(n-1, d)$ by

$$
\Lambda \mapsto(\Lambda \cap E) \subset(V \cap E) .
$$

It again follows from (4.1) that the image of a cell in the shear zone is a cell in the shear zone of a stable manifold of a flow on the image Grassmannian. The rest of the argument is similar.

Proof of the Corollary. $W$ constructed above is nonsingular, and hence its cohomology satisfies Poincaré duality: if $N=\operatorname{dim}(W)$, then $\operatorname{rank}\left(H^{N-i}(W)\right)=$ $\operatorname{rank}\left(H^{i}(W)\right)$. If the cohomology of $X$ does not satisfy Poincaré duality, then there are cells in $X$ not in $W$. These all occur in or below the "shear zone". The complement of $W$ is the singular locus and is nonempty. Q.E.D.

\section{REFERENCES}

[A] Sir Michael Atiyah, Convexity and commuting Hamiltonians, Bull. London Math. Soc. 14 (1982), 1-15.

[B] R. Brockett, Finite dimensional linear systems, Wiley, New York, 1969.

[BB] A. Bialynicki-Birula, Some theorems on the actions of algebraic groups, Ann. of Math. (2) 98 (1973), 480-497.

[D] V. Deodhar, Local Poincaré duality and non-singularity of Schubert varieties, Comm. Algebra 13 (1985), 1379-1388.

[GM] Sergei Gel'fand and R. MacPherson, Verma modules and Schubert cells: a dictionary, Séminaire d'Algèbre Paul Dubreil and Marie-Paul Malliavin, Lecture Notes in Math., vol. 924, Springer-Verlag, New York, 1982.

[HM] M. Hazewinkel and C. F. Martin, Representations of the symmetric group, the specialization order, systems, and the Grassmann manifold, Enseign. Math. 29 (1983), 53-87.

[P] R. Proctor, Classical Bruhat orders and lexicographic shellability, J. Algebra 77 (1982), 104-126. 
[S] M. Shayman, Phase portrait of the matrix Riccati equation, SIAM J. Control Optim. 24 (1986), 1-65.

[W] J. Wolper, A combinatorial approach to the singularities of Schubert varieties, Adv. Math. 76 (1989), 184-193.

Department of Mathematics and Computer Science, Hamilton College, Clinton, New YoRK 13323

Current address: Department of Mathematics, Idaho State University, Pocatello, Idaho 832098085

E-mail address: wolperj@howland.isu.edu 